\title{
Diseño de molde para muñón de pierna con altura ajustable de bajo costo, para prótesis de rodilla con energía cinética
}

\section{Low cost adjustable height leg stump mold design for kinetic energy knee prostheses}

\author{
RIVAS-RODRÍGUEZ, Amando $\dagger$ \\ Tecnológico de Estudios Superiores de Cuautitlán Izcalli, División de ingeniería Industrial. Av. Nopaltepec s/n Fracción La \\ coyotera del ejido San Antonio Cuamatla, C.P. 54748. Cuautitlán Izcalli Estado de México.
}

ID $1^{\mathrm{er}}$ Autor: Amando, Rivas-Rodríguez / ORC ID: 0000-0002-8543-0426, CONACYT CVU ID: 336139

DOI: $10.35429 /$ JOHS.2020.22.7.12.17

Recibido 12 de Enero, 2020; Aceptado 30 de Junio, 2020

\begin{abstract}
Resumen
Objetivo. Diseñar molde y base de muñón de pierna y manufacturarlo, con ajuste de centro de gravedad variable, el costo no debe de superar el $50 \%$ de las prótesis que existen en el mercado, para ello se realizará con materiales accesibles de fácil adquisición y de bajo costo. Metodología. Tomar molde del muñón del sujeto en yeso, posteriormente realizar el macho igual en yeso, para continuar el molde en fibra de vidrio y resina, con un soporte de metal en la parte inferior, donde se acoplará a una base de aluminio maquinado en forma cilíndrica que nos permite ajustar la altura y también un ajuste horizontal, de este modo se ajustará el centro de gravedad, ayudando a la persona tener un mayor equilibrio, y un mejor funcionamiento de la prótesis de rodilla con energía cinética. Contribución: los materiales que se están utilizando se encuentran fácilmente en el mercado, y son de bajo costo, para las personas de bajos recursos que presentan algún problema similar, lo puedan adquirir, y no sea impedimento la falta de una extremidad para continuar con su vida.
\end{abstract}

Prótesis, Costo, Funcional, Diseñar

\begin{abstract}
Objective Design a leg stump mold and base and manufacture it, with variable center of gravity adjustment, the cost should not exceed $50 \%$ of the prostheses that exist in the market, for this it will be made with accessible materials that are easy to acquire and low cost. Methodology Take a mold of the stump of the subject in plaster, then make the male the same in plaster, to continue the mold in fiberglass and resin, with a metal support at the bottom, where it will be attached to a machined aluminum base. Cylindrical that allows us to adjust the height and also a horizontal adjustment, this mode adjusts the center of gravity, helping the person to have a better balance, and a better performance of the knee prosthesis with kinetic energy. Contribution: the materials they are using are easily on the market, and are inexpensive, for low-income people who have a similar problem, what they can get, and there is no impediment to the sea the lack of a limb to continue with his life.
\end{abstract}

Prosthesis, Cost, Functional, Design

Citación: RIVAS-RODRÍGUEZ, Amando. Diseño de molde para muñón de pierna con altura ajustable de bajo costo, para prótesis de rodilla con energía cinética. Revista de Ciencias de la Salud. 2020. 7-22:12-17. 


\section{Introducción}

Las prótesis han servido de gran ayuda a muchas personas a lo largo de la historia. Su evolución, a pesar de haber sido lenta, está llena de misterio y curiosidad debido a la incapacidad de conocer su origen exacto, pero existen investigaciones y rastros que han comprobado que desde hace ya muchos años existen y que han evolucionado con el paso del tiempo y la llegada de las nuevas tecnologías de diseño y elaboración.

Es verdad que con el gran impacto de nuevos productos actualmente podemos contar con una amplia gama de productos en el mercado, esto podría ser de gran beneficio para todos y lo es, pero los costos no se encuentran al alcance de todos, una prótesis de bajo costo aumentaría la posibilidad de que una persona pueda adquirirla.

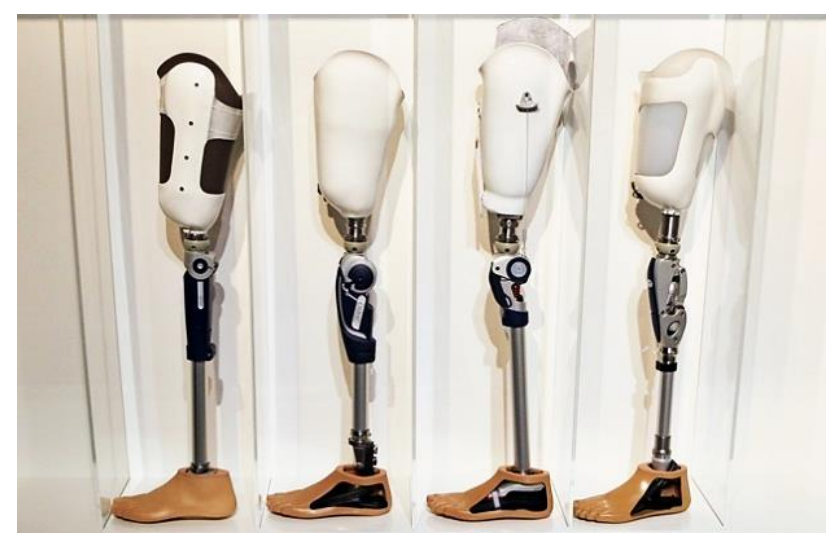

\section{Figura 1 Prótesis}

Fuente:https://zayago.com/conoce-las-mejores-protesisde-pierna-para-diabeticos/

\section{Valor agregado}

Bajo costo, las personas de bajos recursos monetarios podrán comprar y usar esta prótesis, utilizar materiales comerciales como es aluminio, fibra de vidrio, resina, baleros que son fácil de conseguir, ayudando a bajando el costo considerablemente, Mejorar su calidad de vida de una persona con amputación de pierna, al tener libre las manos y poder realizar otras actividades que no sea, el sujetar las muletas.

\section{Características}

Materiales: fibra de vidrio, y aluminio, que se poder adquirir fácilmente, resistentes a la intemperie.
Molde: Fibra de vidrio comúnmente utilizado en el ramo automotriz, con una espiga en la parte inferior que permitirá sujetarla y alinea a la base de sujeción

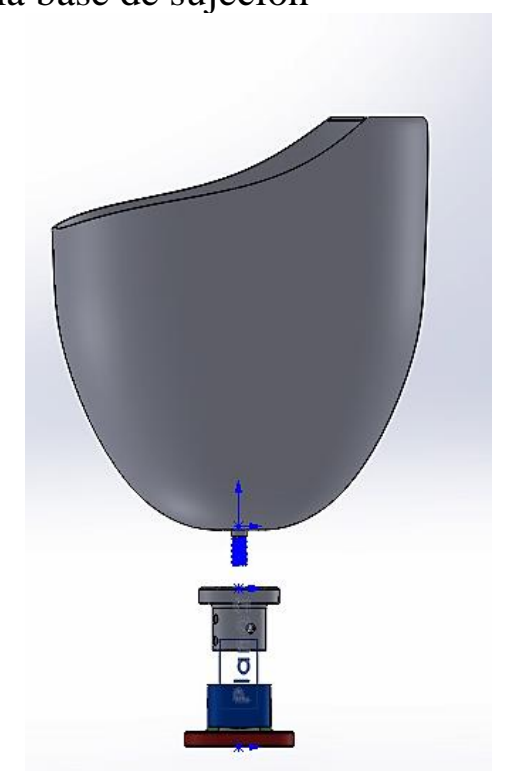

Figura 2 Conjunto de molde y soportes Fuente: Elaboración propia

\section{Diseño}

Base superior de aluminio con una ranura que permite regular el centro de gravedad y ajustarlo, balanceando el peso del usuario hacia adelante ó atrás, mejorando su equilibrio, además de tener oricios que ajustan la altura, e este caso tenemos 3 ajustes de $8 \mathrm{~mm}$. aproximadamente, ensamblándose a distintas alturas, dando mayor comodidad y estabilidad.

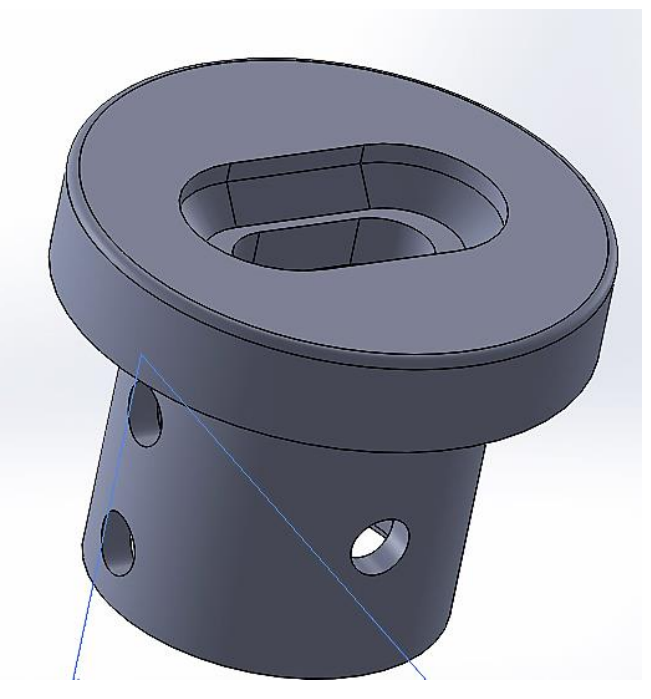

Figura 3 Base superior de molde Fuente: Elaboración propia

Base inferior en aluminio descentrado para desplazar el centro de gravedad, con orificio para su ensamble.

RIVAS-RODRÍGUEZ, Amando. Diseño de molde para muñón de pierna con altura ajustable de bajo costo, para prótesis de rodilla con energía cinética. Revista de Ciencias de la Salud. 2020 


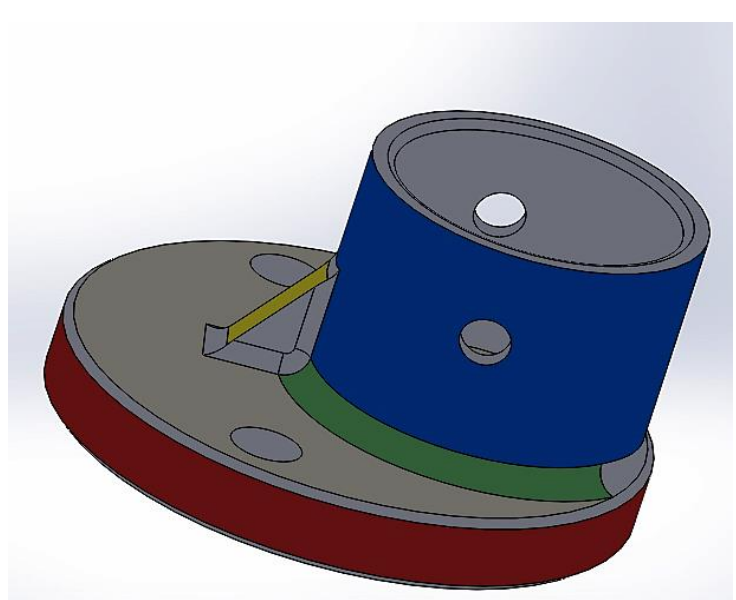

Figura 4 Base inferior de molde

Fuente: Elaboración propia

\section{Planteamiento de problema}

Existen prótesis de costos muy elevados en el mercado y solo una muestra de la población muy pequeña puede adquirirla. En México se amputan 75 personas diarias. Además de los que existen son rígidos es decir están fabricados para una sola dimensión, en el caso de personas adolecentes tiene que remplazar la prótesis cuando aumentes su crecimiento

- Los datos a 2014 muestran que hay cerca de 900 mil amputados en México.

- $\quad$ De 900,000 amputados sólo se rehabilitan 7,500 .

67,500 no llegan a utilizar una prótesis y 5,250 no la saben utilizar "INEGI 2015" (https://www.anmm.org.mx/actas2015/Acta_S O04032015.pdf).

Actualmente se diseñó y manufacturo una prótesis basado en energía cinética, y es necesario acoplarlo al muñón del usuario, pero con ciertas características que ayudaran al buen funcionamiento de la misma, para esto necesitamos un socket del muñón con altura ajustable y poder desplazar el centro de gravedad del usuario ensamblarlo a la prótesis, sin superar el costo de los existentes en el mercado que están en 30 a más 100 mil pesos.

Hipótesis: Es posible Manufacturar una prótesis de altura variable con ajuste en centro de gravedad, que sea compatible para usuarios de diferentes estaturas y complexiones, utilizando materiales convencionales, lograremos bajar los costos en un 50\% total de la prótesis y un nivel de funcionalidad aceptable.

\section{Planteamiento de problema}

Existen prótesis de costos muy elevados en el mercado y solo una muestra de la población muy pequeña puede adquirirla. En México se amputan 75 personas diarias. Además de los que existen son rígidos es decir están fabricados para una sola dimensión, en el caso de personas adolecentes tiene que remplazar la prótesis cuando aumentes su crecimiento

- Los datos a 2014 muestran que hay cerca de 900 mil amputados en México.

- $\quad$ De 900,000 amputados sólo se rehabilitan 7,500 .

67,500 no llegan a utilizar una prótesis y 5,250 no la saben utilizar "INEGI 2015" (https://www.anmm.org.mx/actas2015/Acta_S O04032015.pdf).

Actualmente se diseñó y manufacturo una prótesis basado en energía cinética, y es necesario acoplarlo al muñón del usuario, pero con ciertas características que ayudaran al buen funcionamiento de la misma, para esto necesitamos un socket del muñón con altura ajustable y poder desplazar el centro de gravedad del usuario ensamblarlo a la prótesis, sin superar el costo de los existentes en el mercado que están en 30 a más 100 mil pesos.

Hipótesis: Es posible Manufacturar una prótesis de altura variable con ajuste en centro de gravedad, que sea compatible para usuarios de diferentes estaturas y complexiones, utilizando materiales convencionales, lograremos bajar los costos en un 50\% total de la prótesis y un nivel de funcionalidad aceptable.

Hipótesis: Es posible Manufacturar una prótesis de altura variable con ajuste en centro de gravedad, que sea compatible para usuarios de diferentes estaturas y complexiones, utilizando materiales convencionales, lograremos bajar los costos en un 50\% total de la prótesis y un nivel de funcionalidad aceptable. 


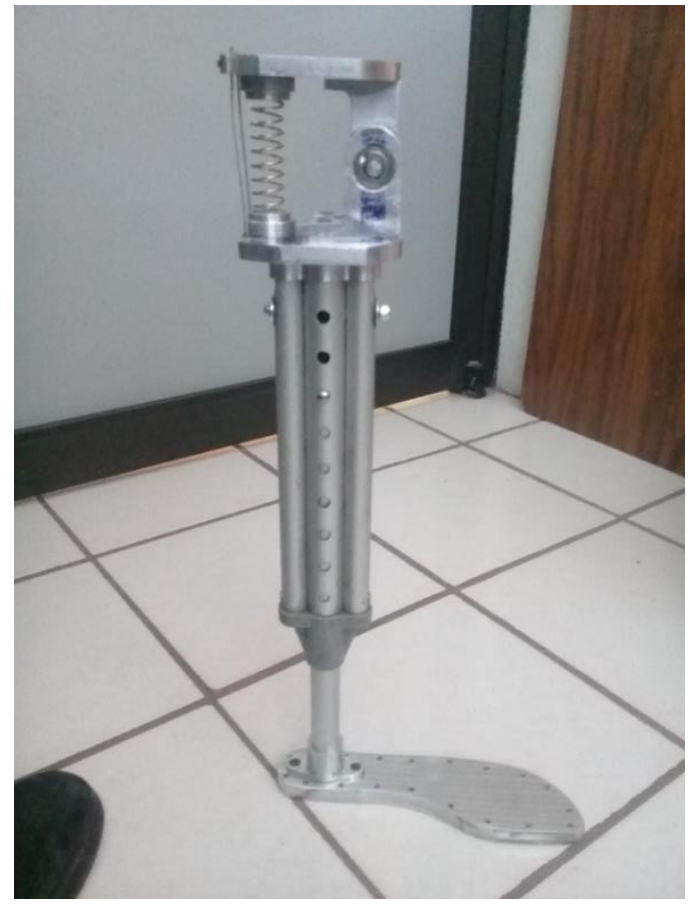

Figura 5 Prótesis para rodilla basada en Energía Cinética Fuente: Elaboración propia

\section{Desarrollo}

Iniciamos con tomar un molde primario al futuro usuario con vendas de yeso, como se muestra en la figura 6 y 7 . Posteriormente se desmoldo y se dio el tiempo de curado, para procer a relizar el macho en yeso.

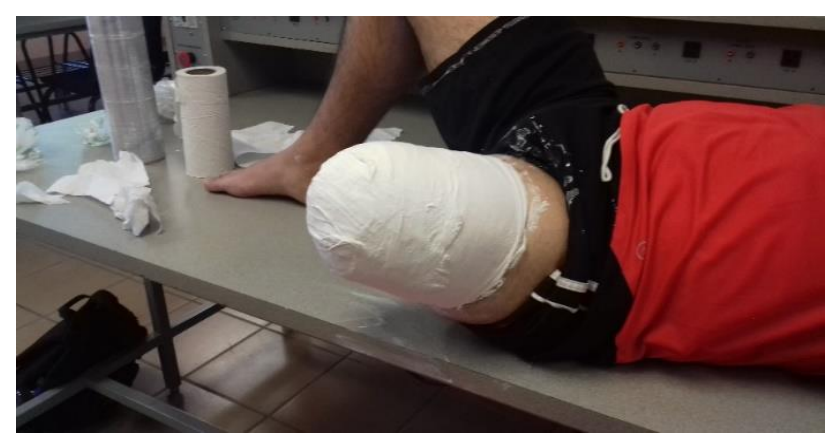

Figura 6 Toma de molde en yeso

Fuente: Elaboración propia

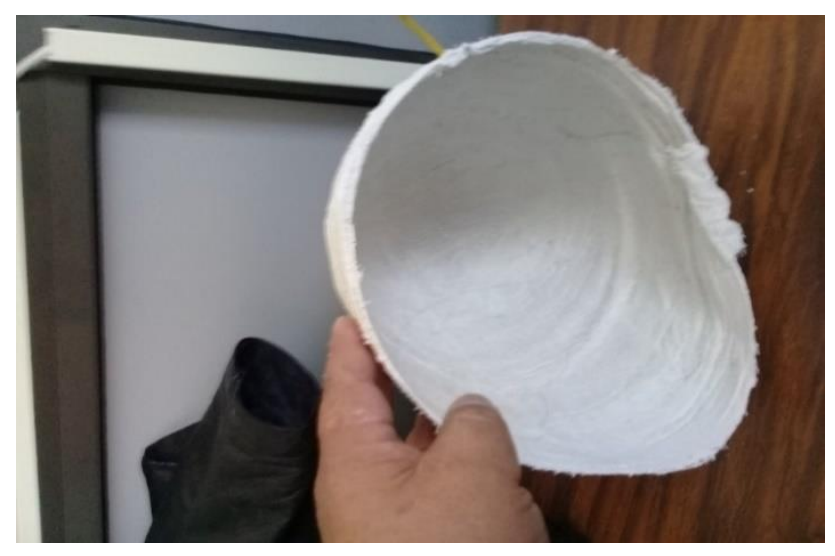

Figura 7 Molde primario en yesso

Fuente: Elaboración propia
Se aplicó una primera capa de resina con fibra de vidrio, y se acoplo base de espiga de acero inoxidable, que previamente se maquino en torno, para posteriormente aplicar una segunda capa de fibra de vidrio y darle el tiempo para su curado, cabe mencionar que se tuvo un primer intento fallido.

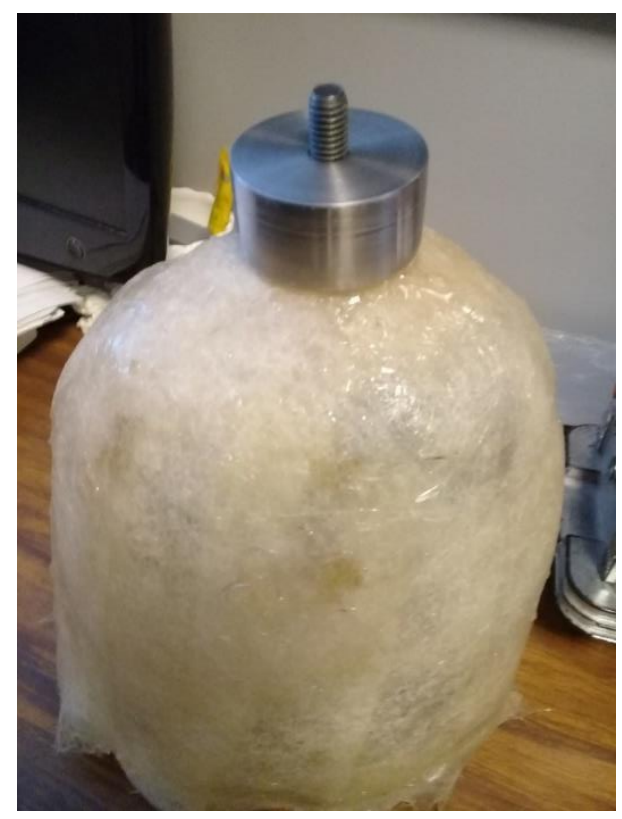

Figura 8 Espiga en molde de fibra de vidrio Fuente: Elaboración propia

Se diseñó las bases, inferior y superior de socket de muñón, para que tengan ajuste tanto en altura como hacia adelante y atrás, esto es lo que dará el ajuste del centro de gravedad, en un primer intento se utilizó una impresora 3D, para su fabricación, con material PLA (figura 8), que fácilmente se fracturo, se imprimió unas segundas piezas en ABS (figura 9) y aunque tuvo una mayor duración se fracturo, por lo que se optó el fabricarlos en aluminio (figura 9).
RIVAS-RODRÍGUEZ, Amando. Diseño de molde para muñón de pierna con altura ajustable de bajo costo, para prótesis de rodilla con energía cinética. Revista de Ciencias de la Salud. 2020 


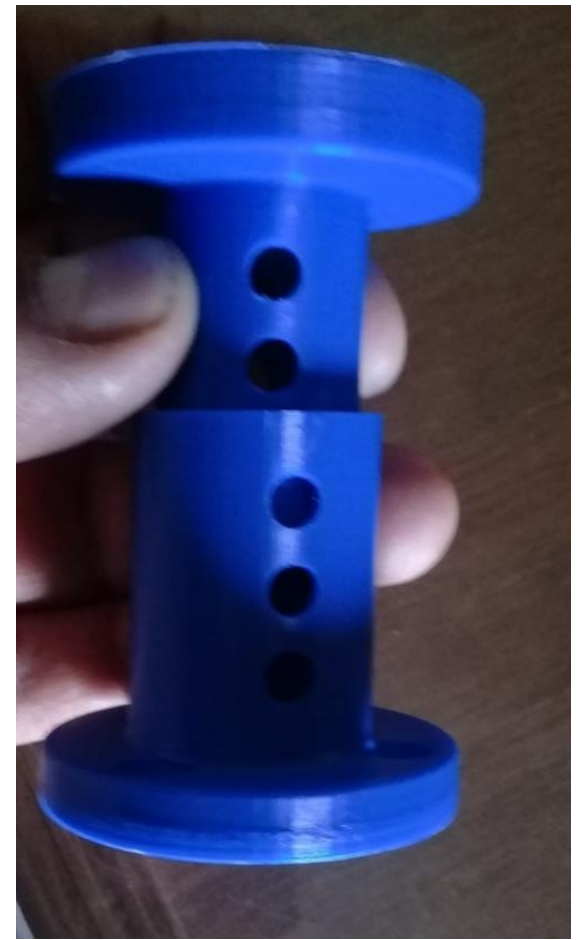

Figura 9 Soportes de molde inferior y superior en PLA Fuente: Elaboración propia

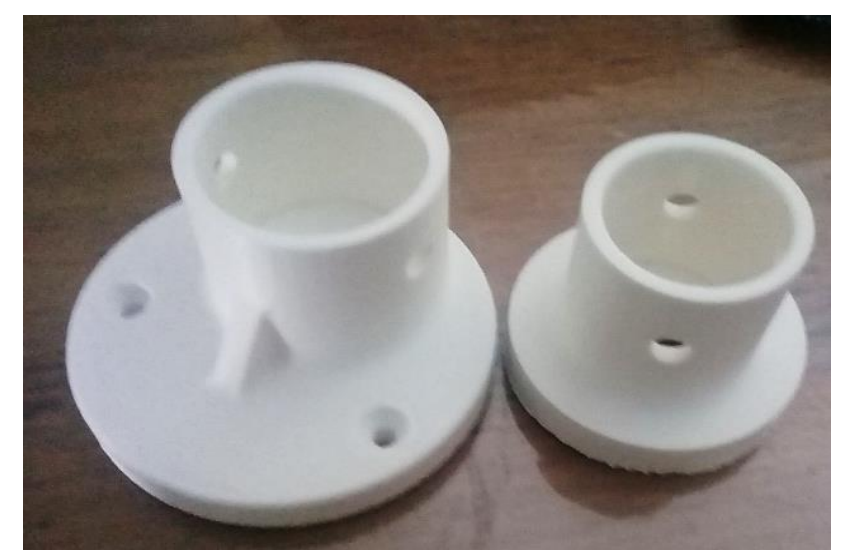

Figura 10 Soportes de molde inferior y superior en ABS Fuente: Elaboración propia

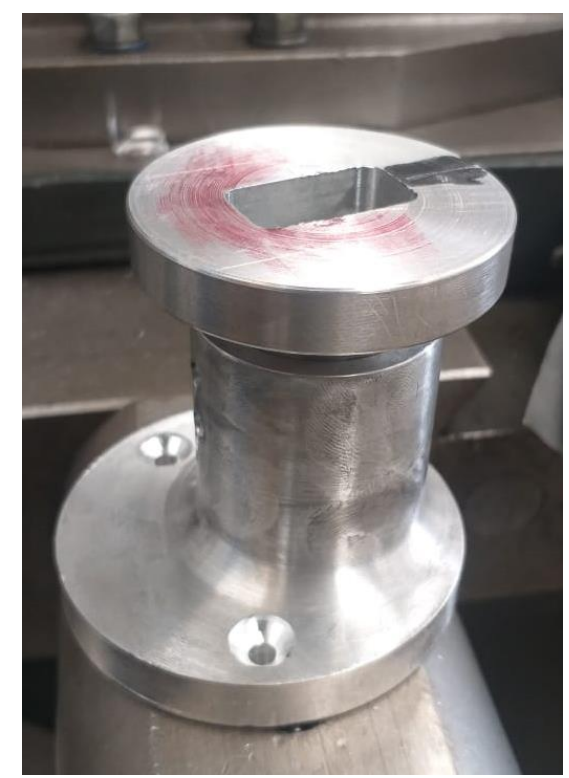

Figura 11 Soportes de molde inferior y superior en aluminio

Fuente: Elaboración propia

ISSN- 2410-3551

ECORFAN® Todos los derechos reservados

\section{Resultados}

Se ensamblar (figura 11) y realizaron las pruebas piloto, los componentes resistiera el esfuerzo y peso, se ajustó centro de gravedad, y el usuario pudo mantenerse de pie sin ayuda de algún soporte, habitualmente utiliza dos muletas.

Para caminar presento algunas dificultades debido a la longitud del molde, se puede apreciar en la figura 11, ocasionando que se saliera del socket por tener poco contacto, esto nos da pie a realizar una mejora en la longitud del muñón, de ese modo aumentar el agarre.

El costo del socket es de aproximadamente $\$ 800$ incluye materiales, como resina, fibra de vidrio barra de aluminio de 3" de diámetro y maquinado del aluminio.

Se continúa trabajando en el nuevo molde de muñón con una longitud más apegada al muñón real.

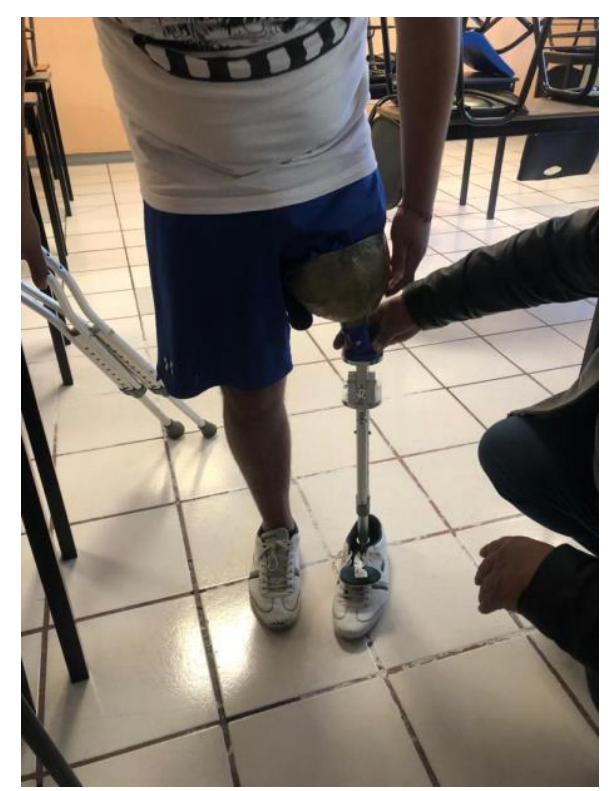

Figura 12 Ensamble de socket y prótesis

Fuente: Elaboración propia

\section{Agradecimientos}

Al alumno Ramírez Rodríguez Julio por prestarse como sujeto de prueba. Al tecnológico de Estudios Superiores de Cuautitlán Izcalli por dar las facilidades e instalaciones para realizar el proyecto. Alumno que apoyaron el proyecto: Villegas López Cesar Eduardo, Salgado Flores Karla Vanessa

Financiamiento: Recursos propios

RIVAS-RODRÍGUEZ, Amando. Diseño de molde para muñón de pierna con altura ajustable de bajo costo, para prótesis de rodilla con energía cinética. Revista de Ciencias de la Salud. 2020 


\section{Conclusiones}

Es factible la manufactura de una prótesis de bajo costo, y centro de gravedad ajustable, el molde del socket del muñón es un componente indispensable, debe de ser amigable con los demás componentes, además que tiene que ser de bajo costo y que sea funcional, para que no aumento el costo total de la prótesis, utilizando materiales que sean fácil de obtener y de remplazar. En esta primera prueba nos da oportunidad de mejorar y optimizar el funcionamiento de la prótesis en conjunto, mejorando el socket de muñón, obtendremos un resultado más favorable.

\section{Referencias}

Alvarado, S., \& Martínez-Hernández, A. (2020). Femoral neck fracture management in patient with bilateral femoral supracondylar amputation and literature review. Acta Ortopédica Mexicana.

Chevalier, A.,(1998), Tecnología del diseño y fabricación de piezas metálicas, Limusa, Noriega.

Gómez, S., (septiembre 2010), SolidWorks Simulation, Alfaomega Grupo Editor S.A. de C.V., México, pp. 480.

Miguel Ángel González Viejo, oriol Cohi Riambau, (2005). Amputacion de extremidad inferior $\mathrm{y}$ discapacidades: protesis $\mathrm{y}$ rehabilitación, Masson.

Villavicencio Barbera, M.F. Diseño de un protocolo para el análisis de requerimientos funcionales en la fabricación de prótesis de pierna bk (below the knee)(Doctoral dissertation) Universidad del Rosario. 